\title{
Influence of the definition of dissipative events on their statistics
}

\author{
E. Buchlin ${ }^{1,2}$, S. Galtier ${ }^{1}$, and M. Velli ${ }^{2,3}$ \\ 1 Institut d'Astrophysique Spatiale, CNRS - Université Paris-Sud, Bât. 121, 91405 Orsay Cedex, France \\ e-mail: eric.buchlin@ias.fr \\ 2 Dipartimento di Astronomia e Scienza dello Spazio, Università di Firenze, 50125 Firenze, Italy \\ ${ }^{3}$ Istituto Nazionale Fisica della Materia, Sezione A, Università di Pisa, 56100 Pisa, Italy
}

Received 12 November 2004 / Accepted 7 February 2005

\begin{abstract}
A convenient and widely used method to study the turbulent plasma in the solar corona is to examine statistics of properties of events (or structures) associated to flares either in observations or in numerical simulations. Numerous papers have followed such a methodology, using different definitions of an event, but the reasons behind the choice of a particular definition is very rarely discussed. We give here a comprehensive set of possible event definitions starting from a one-dimensional data set such as a time-series of energy dissipation. Each definition is then applied to a time-series of energy dissipation obtained from simulations of a shell-model of magnetohydrodynamic turbulence, or from a new model of coupled shell-models designed to represent a magnetic loop in the solar corona. We obtain distributions of the peak dissipation power, total energy, duration and waiting-time associated with each definition. These distributions are then investigated and compared, and the influence of the definition of an event on the statistics is discussed. In particular, power-law distributions are more likely to appear when using a threshold. The sensitivity of the distributions to the definition of an event seems also to be weaker for events found in a highly intermittent time series. Some implications for statistical results obtained from observations are discussed.
\end{abstract}

Key words. Sun: corona - Sun: flares - magnetohydrodynamics (MHD) - methods: data analysis

\section{Introduction}

The mechanism heating the solar corona to millions of degrees remains an open problem, but it is generally understood that a great part of the energy dissipation must occur at scales that are smaller than the structures that can be resolved by observations $(\approx 100 \mathrm{~km})$, as small as $10-100 \mathrm{~m}$ (the Kolmogorov turbulent cascade dissipation scale). One of the most successful approaches to reach this four-order-of-magnitude wide gap is to assume that the statistics obtained at observable scales are still valid at smallest scales. The properties of the global system, from observable to non-observable scales, can then be investigated. For example this is the idea underlying Hudson's (1991) critical power-law slope of -2 for the distribution of flare energies.

The measurement of the power-law slope for the lowest energy flares has been a major goal of coronal physics in the last decade. Aschwanden et al. (2000) has summarized the distributions of event energies that were obtained at wavelengths from $\mathrm{X}$-rays to ultra-violet (UV), and for event energies covering a range of eight orders of magnitude from $10^{17} \mathrm{~J}$ ("nanoflares") to $10^{25} \mathrm{~J}$ ("flares"). It seems - as asserted by Aschwanden et al. (2000) - that these distributions can be matched to form a unique power-law distribution of slope $\approx-1.8$.

However, for the smallest events, mainly observed in UV by filter imaging instruments, some observations seem not to mutually agree, and they seem not to fit in the global distribution. A possible explanation for this is given by Aschwanden \& Charbonneau (2002): the energy of an event could be wrongly deduced from the observable quantities (like intensity in some spectral lines), leading to a systematic error in the distribution of event energies. Another explanation could be that all authors do not agree on what they mean by "event", i.e. the fact that non-equivalent definitions exist in the literature.

Indeed, defining an event is likely to be much more difficult for low energy events than for high energy events. For high-energy events, whose distributions are in general derived from X-rays and radio observations, there is little ambiguity as to what constitutes an event: events are very rare $\left(10^{-6} \mathrm{~s}^{-1}\right.$ for the whole solar disk between $10^{23}$ and $10^{24} \mathrm{~J}$ ) and are wellseparated by long low-flux times. On the contrary, low-energy events can be very close in space and time, making it difficult to separate them, either because they occur on the same lineof-sight or because they are smaller than the instrumental resolution or shorter than the time resolution. The difficulty is even greater if we subscribe to the idea that the corona is in a selforganized critical state so that small events trigger other events, leading to avalanches as illustrated by the sandpile paradigm (Bak et al. 1988; Lu \& Hamilton 1991): among all these events, which ones should be used to determine the statistics?

We think that this difficulty has been underestimated when statistics obtained from observations or simulations by 
different methods have been compared. The definition of an event used is very rarely discussed (contrary to the influence of the relationship between the observable parameters and the physical variables of an event), and is sometimes not even given precisely.

In this paper we give some definitions that could be used, mainly inspired from definitions that have already been used in the past. We choose to restrain ourselves to events defined from a one-dimensional data set, namely a time series of energy dissipation, so that the definitions can be easily compared. We then produce statistics of events (histograms of event energies, durations and waiting times), for different definitions of an event, and compare them. The time series we use are the data output by a shell-model of MHD turbulence (Giuliani \& Carbone 1998), and by a new model of coupled shell-models describing Alfvén turbulence in a coronal loop (Buchlin et al. 2004). However, the aim of this paper is not to study shellmodels of MHD, but to determine to which extent the definition of events influences their statistics, even in a simple case of events detected in a time series of energy dissipation.

\section{Event definitions}

We present here a basic list of possible definitions of an event when a "signal" $\epsilon(t)$, which is the time series of the power dissipated in the system, is given (Fig. 1). Most of the ideas of this list come from the definitions that have been chosen in papers found in the literature. For each event, we get:

- $E$, the total energy dissipated during the event;

- $P$, the peak power of energy dissipation;

- $T$, the duration of the event;

$-t_{\mathrm{e}}$, the time of the event, necessary to obtain the waiting times $\tau_{\mathrm{w}}$, i.e. the (quiescent) time between two consecutive events.

\subsection{Peaks}

Definition 1 (peak). An event corresponds to a local maximum $\epsilon\left(t_{\mathrm{m}}\right)$ in the signal $\epsilon(t)$. The time of the event is $t_{\mathrm{e}}=t_{\mathrm{m}}$, the peak dissipation power is $P=\epsilon\left(t_{\mathrm{e}}\right)$, the total dissipated energy is $E=\int_{t_{b}}^{t_{b}} \epsilon(t) \mathrm{d} t$ where $t_{a}$ and $t_{b}$ are the two local minima around $t_{\mathrm{e}}$, and the event duration is $T=t_{b}-t_{a}$.

Variant 1.1 (peak-background). The background $b(t)$ is the affine function defined between the points $\left(t_{a}, \epsilon\left(t_{a}\right)\right)$ and $\left(t_{b}, \epsilon\left(t_{b}\right)\right)$. With the notations of definition 1 , the time of the event is $t_{\mathrm{e}}$, the peak dissipation power is $P-b\left(t_{\mathrm{e}}\right)$, the total dissipated energy is $E-\int_{t_{a}}^{t_{b}} b(t) \mathrm{d} t=E-\left(\epsilon\left(t_{a}\right)+\epsilon\left(t_{b}\right)\right) \cdot T / 2$, and the event duration is $T$.

\subsection{Threshold}

Definition 2 (threshold). A threshold $\epsilon_{\mathrm{thr}}$ is chosen, and an event is a part of the signal $\epsilon(t)$ which stays above $\epsilon_{\mathrm{thr}}$ : more precisely, it is a maximal connex part $V=\left[t_{a}, t_{b}\right]$ of the set $\left\{t \mid \epsilon(t)>\epsilon_{\mathrm{thr}}\right\}$. The total dissipated energy is $E=\int_{V} \epsilon(t) \mathrm{d} t$, the peak dissipation power is $P=\max _{V} \epsilon(t)$ and the event duration is $T=t_{b}-t_{a}$. The time of the event is the time at which the maximum of $\epsilon(t)$ on $V$ is attained: $\epsilon\left(t_{\mathrm{e}}\right)=\max _{V} \epsilon(t)$.

There are several other alternatives to define the time of the event, like $t_{\mathrm{e}}=\left(t_{b}+t_{a}\right) / 2$ (the middle of interval $V$ ) or $t_{\mathrm{e}}=\frac{1}{E} \int_{V} \epsilon(t) \cdot t \mathrm{~d} t$ (the barycenter of the event, weighted by $\epsilon$ ). But these variants do not change the statistics of $P, E$, and $T$, and they seem to have little influence on the statistics of $\tau_{\mathrm{w}}$.

We can consider the threshold level $\epsilon_{\mathrm{thr}}$ as a background level, giving the following definition:

Variant 2.1 (threshold-background). Using definition 2 and its notations, the time of the event is $t_{\mathrm{e}}$, the peak dissipation power is $P-\epsilon_{\mathrm{thr}}$, the total dissipated energy is $E-\int_{V} \epsilon_{\mathrm{thr}} \mathrm{d} t=$ $E-\epsilon_{\mathrm{thr}} \cdot T$, and the event duration is $T$.

The threshold can be chosen as a function of the overall average $\bar{\epsilon}$ and standard deviation $\sigma_{\mathrm{e}}$ of $\epsilon(t)$. It can also be chosen iteratively, by using the average and standard deviation of the time series during the quiescent times between events (which have been defined by the previous iteration of this process), as in Boffetta et al. (1999).

\subsection{Wavelet analysis}

This method assumes that we have built the time-scale plane $y\left(t_{0}, s\right)$ for $\epsilon(t)$, by convolution of $\epsilon(t)$ by the wavelets $w_{t_{0}, s}(t)=$ $1 / s \cdot w_{0}\left(\left(t-t_{0}\right) / s\right)$. A mother wavelet $w_{0}$ which seems well adapted to the shape of events is the second derivative of a Gaussian ("Mexican hat"). When the noise is in $1 / f$, Sanz et al. (2001) have shown that the Mexican hat is the best wavelet to find enhancements of the signal.

Definition 3 (wavelet). An event corresponds to a local maximum $y\left(t_{\mathrm{e}}, s_{\mathrm{e}}\right)$ in the time-scale plane $y\left(t_{0}, s\right)$. The time of the event is $t_{\mathrm{e}}$, its duration $T$ is the scale $s_{\mathrm{e}}$, its total energy is $E=y\left(t_{\mathrm{e}}, s_{\mathrm{e}}\right)$. Its peak power $P$ can be defined as $\max _{V} \epsilon(t)$ with $V=\left[t_{\mathrm{e}}-s_{\mathrm{e}} / 2, t_{\mathrm{e}}+s_{\mathrm{e}} / 2\right]$.

For better accuracy in the definition of $s_{\mathrm{e}}$ and $t_{\mathrm{e}}$, we need to have a good resolution in the time-frequency plane, i.e. we use a continuous wavelet transform. As a result, and also because we have used the Mexican hat wavelet, the wavelets are non-orthogonal, and there is a risk of finding several nonindependent events where there is only one event. To avoid this, it is possible to impose that two events are separated by at least some distance in the time-scale plane (typically a factor of 2 in scale and an interval $s_{\mathrm{e}}$ in time), but in practice this is not necessary as the time-scale plane is sufficiently smooth.

\section{Model time series}

\subsection{Shell-model}

The results of the next section are based on data output from a shell-model of incompressible MHD turbulence. In such models (Gloaguen et al. 1985; Biskamp 1994; Frick \& Sokoloff 1998; Giuliani \& Carbone 1998), the Fourier wavenumber space is divided in concentric shells $S_{n}=\left\{\boldsymbol{k} \mid\|\boldsymbol{k}\| \in\left[k_{n}, k_{n+1}\right]\right\}$ 

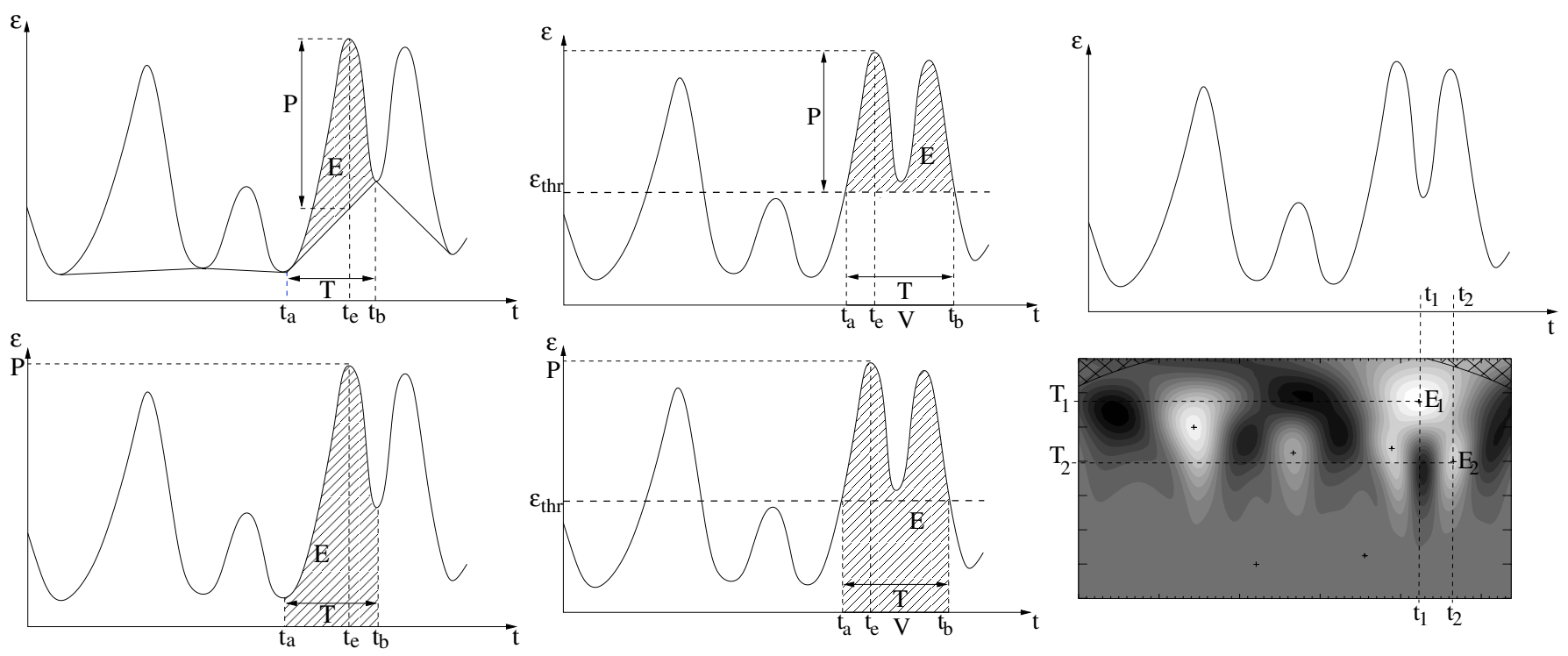

Fig. 1. Definitions of events and of event characteristics $\left(t_{\mathrm{e}}, T, P\right.$, and $\left.E\right)$. Left: peaks (definition 1), with (top) and without (bottom) background detection. Middle: threshold (definition 2), with (top) or without (bottom) taking the background into account. Right: peaks in the wavelets time-frequency plane (definition 3). For this definition, events are marked as crosses in the time-frequency plane, indicating the event time $t_{\mathrm{e}}$ and duration $T$. The total energy $E$ is the wavelet amplitude (gray level) at this position.

with $k_{n}=k_{0} \lambda^{n}$ and usually $\lambda=2$. A single complex scalar value $u_{n}$ represents the velocity increments $|u(x+\ell)-u(x)|$ on scales $\ell$ for $2 \pi / \ell \in S_{n}$. The same holds for the scalar value $b_{n}$ representing the magnetic field increments on the same scales $\ell$. This model is the magnetohydrodynamic analog of the GOY (Gledzer-Ohkitani-Yamada: Gledzer 1973; Yamada \& Ohkitani 1987, 1988a,b) shell-model of fluid turbulence. It is governed by the following equations, given in Giuliani \& Carbone (1998):

$\frac{\mathrm{d} Z_{n}^{s}}{\mathrm{~d} t}=-k_{n}^{2}\left(v^{+} Z_{n}^{s}+v^{-} Z_{n}^{-s}\right)+\mathrm{i} k_{n} T_{n}^{s *}+f_{n}^{s}$

where $Z_{n}^{s}=v_{n}+s b_{n}$ are the Elsässer variables, $s= \pm$, $v^{s}=(v+s \eta) / 2$ are combinations of kinematic viscosity and resistivity, $f_{n}^{s}$ are external driving forces, and $T_{n}^{s}$ is the term corresponding to local non-linear interactions between shells. For a given shell $n$, this term involves the neighbors and secondnearest neighbors of the shell $n$, modelling local interactions between triads of consecutive modes. The detailed coefficients of this term are given in Giuliani \& Carbone (1998) and depend on the dimensionality (e.g. 2D or 3D) of the physical MHD system the shell-model represents, via the conservation of the MHD invariants.

This model can describe the evolution of modes over a wide range of wavenumbers with just a few dozen degrees of freedom. It is thus very interesting for studying MHD turbulence with high Reynolds numbers, and intermittency. It exhibits typical properties of MHD turbulence, from the dynamo effect in 3D to wide power-law spectra (Giuliani \& Carbone 1998), including spatial and temporal intermittency (Giuliani \& Carbone 1998; Boffetta et al. 2002).

The equations 1 are solved numerically and we get the time series of dissipated power $\epsilon(t)=\sum_{n} k_{n}^{2}\left(v\left|u_{n}(t)\right|^{2}+\eta\left|b_{n}(t)\right|^{2}\right)$, which is our variable of interest. To obtain the first time series shown in Fig. 2, hereafter known as the time series $\langle 1\rangle$, we used 24 shells (representing $k=1$ to $k \approx 8.4 \times 10^{6}$ ), with $\lambda=2$, $v=\eta=10^{-11}$. We performed $10^{7}$ variable timesteps (determined by a CFL condition) with a 3rd-order Runge-Kutta numerical scheme. The time series $\langle 2\rangle$, also shown in Fig. 2, was obtained with the same parameters, except that the dissipation coefficients $v$ and $\eta$ where ten times higher than for $\langle 1\rangle$.

\subsection{Coupled shell-models}

Section 5 also uses data from a version of a shell-model designed to model a region of space where a dominant magnetic field $\boldsymbol{B}_{0}$ exists, like in a coronal loop (Buchlin et al. 2004). In this model, shell-models of 2D MHD are coupled by Alfvén waves travelling along $\boldsymbol{B}_{0}$, and energy is only input by movements of the photospheric footpoints of the loop. This geometric setup is the same as the one used for the cellular automaton described by Buchlin et al. (2003), and it gives a model similar to the one described by Nigro et al. (2004). Here we use an independent implementation of these ideas to obtain the time series $\langle 3\rangle$.

\subsection{Characteristics and intermittency of the time series}

All these time series were rescaled so that their average $\bar{\epsilon}$ is 1 and are shown on Fig. 2. Their basic characteristics are summarized in Table 1. From time series $\langle 1\rangle$ to $\langle 3\rangle$, the ratio of the maximum (or the standard deviation) to the average grows, and longer quiet times exist between the intervals with higher dissipation. It seems that intermittency is higher for $\langle 3\rangle$ than for $\langle 2\rangle$, and that it is also higher for $\langle 2\rangle$ than for $\langle 1\rangle$. This is verified by 

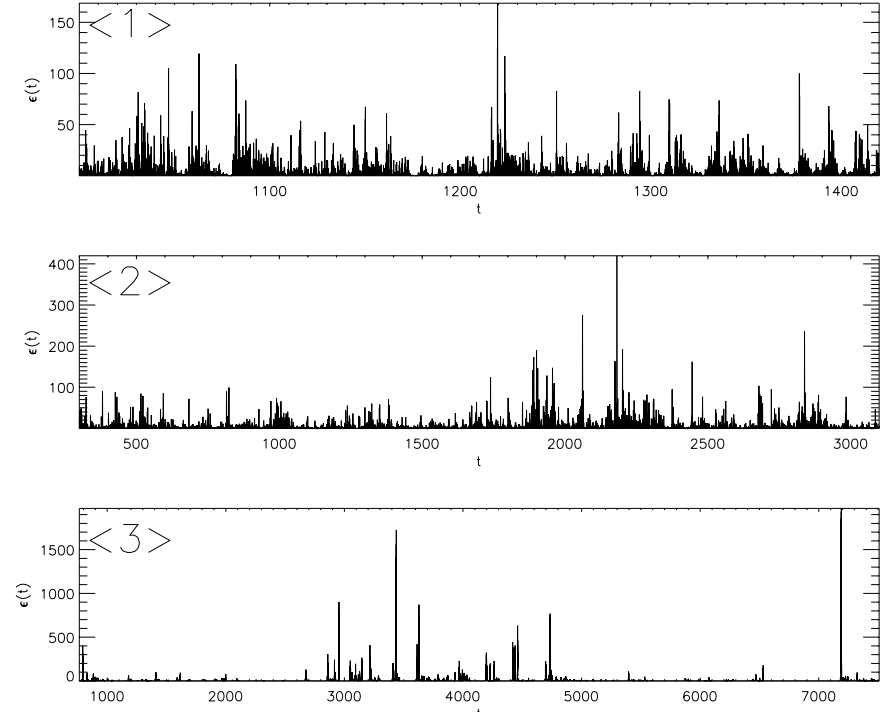

Fig. 2. From top to bottom, time series $\langle 1\rangle,\langle 2\rangle$, and $\langle 3\rangle$.

Table 1. Summary of the characteristics of all 3 time series, which were normalized so that their average is $\bar{\epsilon}=1$ : number of data points, number of peaks, standard deviation, and maximum value.

\begin{tabular}{ccccc}
\hline \hline & Data points & Peaks & $\sigma_{\epsilon}$ & $\epsilon_{\max }$ \\
\hline$\langle 1\rangle$ & 453628 & 51507 & 1.98 & 169 \\
$\langle 2\rangle$ & 985162 & 56136 & 2.45 & 420 \\
$\langle 3\rangle$ & 1000000 & 305738 & 11.33 & 1971 \\
\hline
\end{tabular}

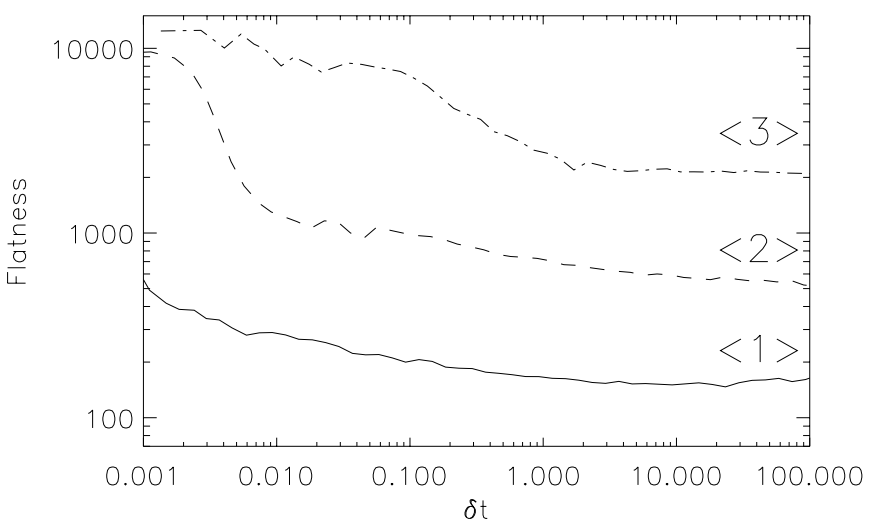

Fig. 3. The flatness of the three time series increases when the scale $\delta t$ decreases. This behavior is a signature of intermittency, and is stronger for the time series $\langle 3\rangle$ and lower for time series $\langle 1\rangle$. Note that the flatness remains much higher than the Gaussian value 3 even at large time scales, as a consequence of the non-Gaussian distribution function of the values taken by the time series.

plotting the flatness ${ }^{1}$ of these time series as a function of the temporal scale (Fig. 3).

\footnotetext{
${ }^{1}$ We use the following definition for the flatness $F(\tau)$ of the time series $\epsilon(t): F(\tau)=S^{4}(\tau) /\left(S^{2}(\tau)\right)^{2}$, where $S^{q}(\tau)=\left\langle|\epsilon(t+\tau)-\epsilon(t)|^{q}\right\rangle_{t}$ is the structure function of index $q$ for the timeseries $\epsilon$.
}
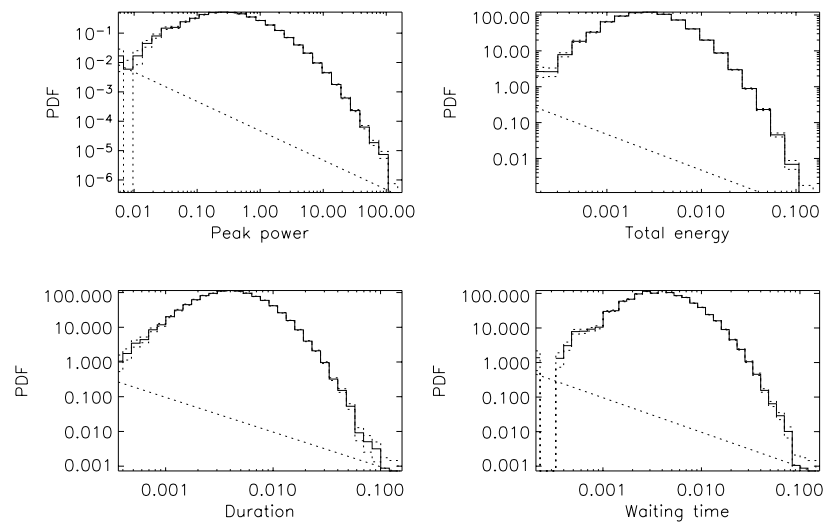

Fig. 4. Events statistics of time series $\langle 1\rangle$ for definition 1 (peaks): peak power of energy dissipation, dissipated energy, duration, and waiting times. The straight dashed line corresponds to one event per histogram bar; as the histogram bars are spaced exponentially, its slope is -1 . The dashed histograms are an estimation of the discretization error when building the histogram, computed assuming Poisson statistics in each $\operatorname{bar}( \pm \sqrt{N}$ where $N$ is the number of events in a given histogram bar).

\section{Comparison between statistics for different event definitions}

In this section, starting from the time series $\epsilon(t)$ number $\langle 1\rangle$ produced as explained in Sect. 3.1, we compare the effect of the definition of an event for the following statistics:

- normalized histograms (i.e. experimental Probability Distribution Functions - or PDFs) of event peak dissipation power, total energy and duration, as defined by the different event definitions we use,

- PDFs of waiting times between events, i.e. the time between two successive events. This corresponds to the laminar, quiet time between events.

\subsection{Peaks}

As each peak of the time series is counted as an event, definitions 1 and 1.1 give many events, even in the case of our numerical data, which has no noise: for time series $\langle 1\rangle$ for example, one data point out of nine is a local maximum, and corresponds thus to an event. When noise is present, a smoothening of the data at the scale of the shortest events may be necessary before searching for events. Furthermore, the set of the events is a partition of the time series (the end $t_{b}$ of one event is the beginning $t_{a}$ of the next event), all the energy of the time series is contained in the events: $\sum_{i} E_{i}=\int \epsilon(t) \mathrm{d} t$.

The distributions of $P, E, T$ and $\tau_{\mathrm{w}}$ (Fig. 4) have approximately the same shape, which is neither a power-law, nor an exponential or Gaussian. The tail of the waiting-time distribution could even be fitted by an exponential (Fig. 5), in contradiction to previous studies of shell-models, which used another definition of an event (Boffetta et al. 1999; Lepreti et al. 2001).

With these definitions, even the smallest peaks are counted as events, and this breaks the waiting times into small parts, leading to a cut-off of the tail of the waiting-time distribution. To decrease this effect, we may exclude the smallest events (e.g. those with a peak power lower than a given 


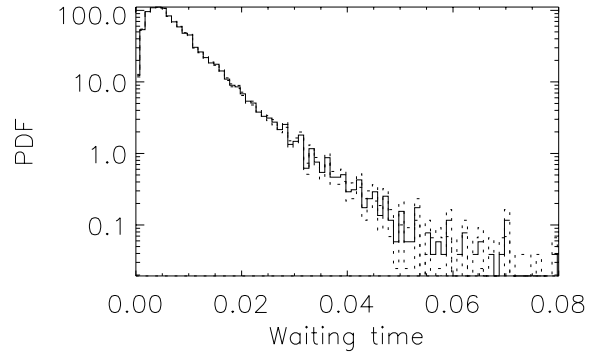

Fig. 5. Waiting-time distribution of events from time series $\langle 1\rangle$ for definition 1 (peaks), in lin-log scale.
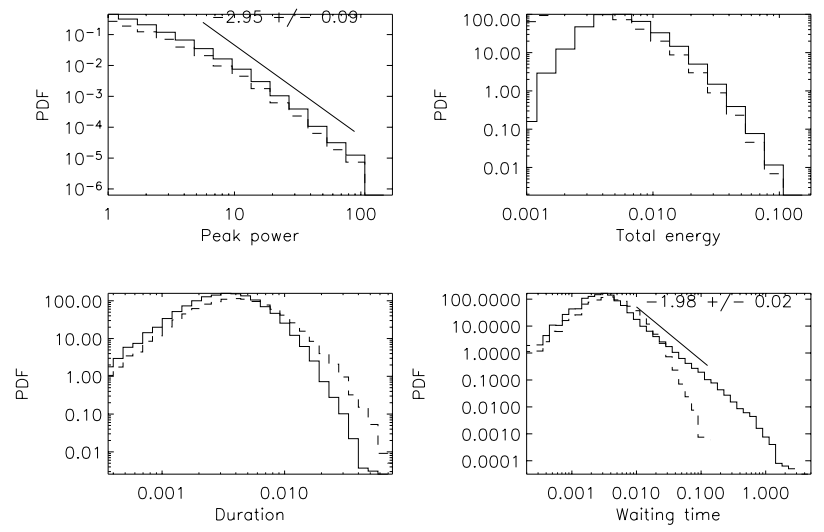

Fig. 6. Events statistics of time series $\langle 1\rangle$ for definition 1.2 (peakthreshold) with $\epsilon_{\mathrm{thr}}=1$ (average of time series). The statistics for definition 1 (peaks) are shown as a reference, in dashed lines.

threshold) from the analysis, which gives the following variant of definition 1:

Variant 1.2 (peak-threshold). A threshold $\epsilon_{\mathrm{thr}}$ is chosen. An event corresponds to a local maximum $\epsilon\left(t_{\mathrm{m}}\right)$ in the signal $\epsilon(t)$, provided that $\epsilon\left(t_{\mathrm{m}}\right)>\epsilon_{\mathrm{thr}}$. The time of the event is $t_{\mathrm{e}}=t_{\mathrm{m}}$, the peak dissipation power is $P=\epsilon\left(t_{\mathrm{e}}\right)$, the total dissipated energy is $E=\int_{t_{a}}^{t_{b}} \epsilon(t) \mathrm{d} t$ where $t_{a}$ and $t_{b}$ are the two local minima around $t_{\mathrm{e}}$, and the event duration is $T=t_{b}-t_{a}$.

Note that this is not the same as using definition 2: for a given threshold $\epsilon_{\mathrm{thr}}$, on a maximum connex part $V$ of $\{t \mid \epsilon(t)>$ $\left.\epsilon_{\text {thr }}\right\}$, definition 1.2 will find as many events as there are peaks of $\epsilon(t)$ in the interval $V$, whereas definition 2 will find only one event.

As a result (Fig. 6), it is clear that the PDF of $P$ is cut below the value of $\epsilon_{\mathrm{thr}}$, with no modification of its shape: this means that only the tail $P>\epsilon_{\text {thr }}=1$ of the histogram of $P$ in Fig. 4 is left, and this tail could be fitted to a quite narrow power-law of slope -2.95 . The PDFs of $E$ and $D$ do not change dramatically, except that the left part is weaker because of the correlations between $P, E$ and $D$. The most interesting effect of using variant 1.2 instead of definition 1 is on the waiting-time distribution: it now exhibits a clear power-law of index -1.98 over 2.5 decades. This is made possible by the fact that small waiting times associated with small events in the case of definition 1 are now replaced by a smaller number of long waiting times, leading to a reinforcement of the right part of the histogram of $\tau_{\mathrm{w}}$.
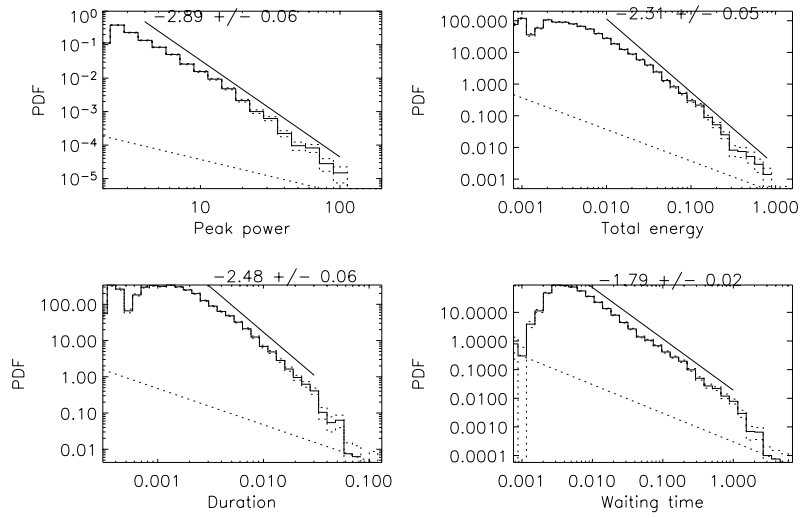

Fig. 7. Event statistics of time series $\langle 1\rangle$ for definition 2 (threshold).
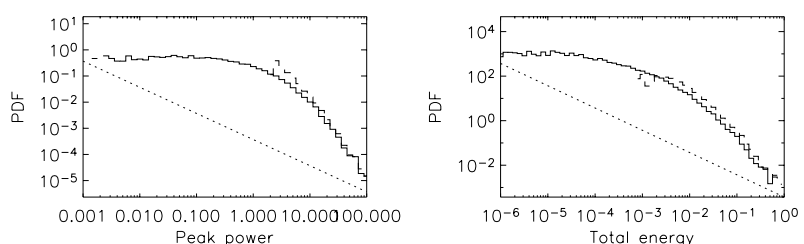

Fig. 8. Event statistics of time series $\langle 1\rangle$ for definition 2.1 (thresholdbackground). Only the distributions that are different from the one obtained by definition 2 (threshold, Fig. 7) are represented.

\subsection{Threshold}

With definition 2, histograms of $P, E, T$, and $\tau_{\mathrm{w}}$ are quite clearly power-laws (Fig. 7), even if they are not very wide for this weakly intermittent time series. The slopes of these powerlaws are $2.89 \pm 0.06$ for $P, 2.31 \pm 0.05$ for $E, 2.48 \pm 0.06$ for $T$, and $1.79 \pm 0.02$ for $\tau_{\mathrm{w}}$.

These power-law tails still exist when the threshold is considered as a background and is removed (variant 2.1, Fig. 8), but the left part of the histograms is then almost flat on logarithmic axes. This is for example quite straight-forward for the distribution of $P$, as removing the background shifts - in linear axes - the distribution of $P$ to the left. However, the right tail of the distributions, e.g. for $P \gg \epsilon_{\mathrm{thr}}$ remains almost the same when the background is removed. It seems that background removal does not help us to understand the statistics of events.

Methods using a threshold are very widely used when events are searched for in time series, as well as from numerical simulations: Dmitruk et al. (1998); Einaudi et al. (1996); Georgoulis et al. (1998) (2D RMHD), Boffetta et al. (1999) (MHD shell-models) and X-rays observations: Pearce et al. (1993); Crosby et al. (1993); Wheatland et al. (1998). It seems to be well-adapted to instrumental constraints of sensitivity and noise levels.

The drawbacks of this definition are that it misses the lowest-energy events (leading to a cut-off of the left part of the energy histograms), and that it cannot separate close highenergy events. This definition is also not adapted to nonstationary time series: in this case, the threshold should adapt to the local statistical characteristics of the time series. 

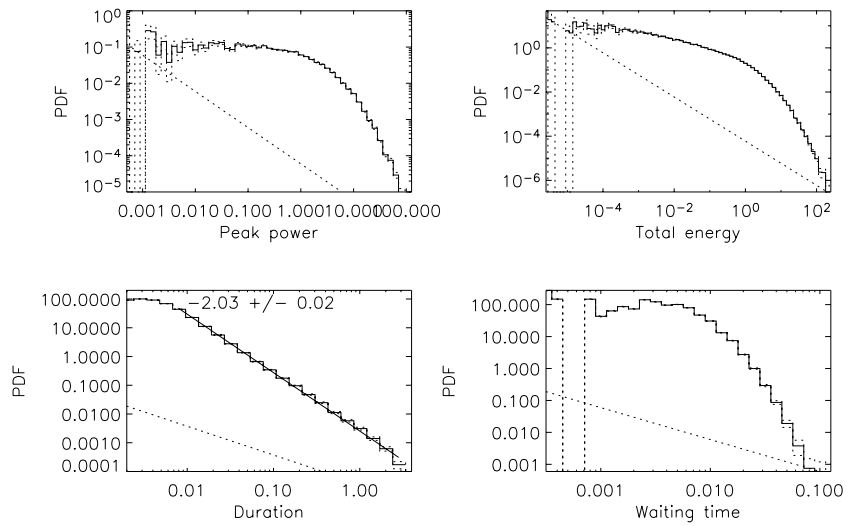

Fig. 9. Event statistics of time series $\langle 1\rangle$ for definition 3 (maxima in wavelet time-scale space).
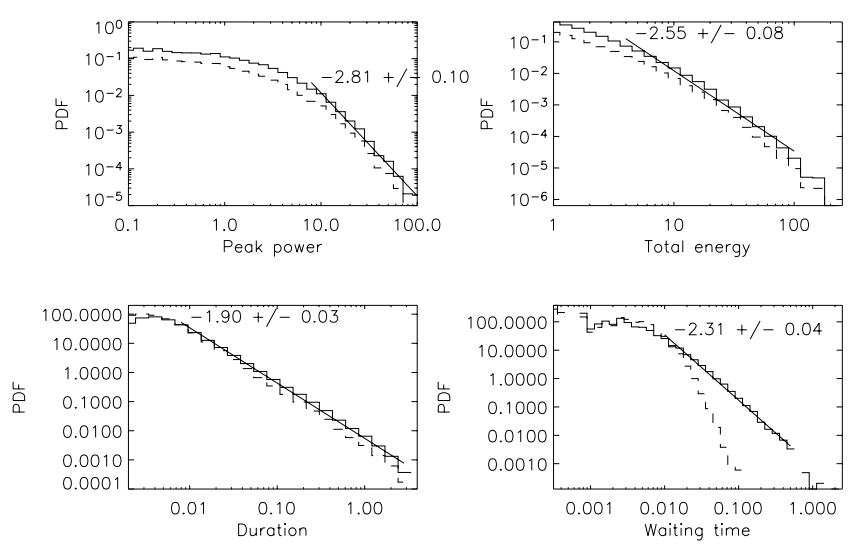

Fig. 10. Event statistics of time series $\langle 1\rangle$ for definition 3.1 (waveletthreshold) and $E_{\mathrm{thr}}=1$. The statistics for definition 3 (maxima in wavelet time-scale space) are shown as a reference, in dashed lines.

\subsection{Wavelet analysis}

Definition 3 produces the histograms of $P, E, D$, and $\tau_{\mathrm{w}}$ shown in Fig. 9. The histogram of event durations is a power-law over more than 2.5 decades. The wide and flat left part of the histograms of $P$ and $E$, which includes events much smaller than with other definitions, suggests use of a variant of definition 3 similar to variant 1.2 of definition 1 , where the smallest events are simply not taken into account:

Variant 3.1 (wavelet-threshold). A threshold $E_{\text {thr }}$ is chosen. An event corresponds to a local maximum $y\left(t_{\mathrm{e}}, s_{\mathrm{e}}\right)$ in the timescale plane $y\left(t_{0}, s\right)$, provided that $y\left(t_{\mathrm{e}}, s_{\mathrm{e}}\right)>E_{\mathrm{thr}}$. The time of the event is $t_{\mathrm{e}}$, its duration $T$ is the scale $s_{\mathrm{e}}$, its total energy is $E=y\left(t_{\mathrm{e}}, s_{\mathrm{e}}\right)$. Its peak power $P$ can be defined as $\max _{V} \epsilon(t)$ with $V=\left[t_{\mathrm{e}}-s_{\mathrm{e}} / 2, t_{\mathrm{e}}+s_{\mathrm{e}} / 2\right]$

As for definition 1.2 (peak-threshold), the distributions of $P, E$ and $D$ do not change much, but a power-law is recovered (Fig. 10) for the waiting-time distribution.

\section{Intermittency and sensitivity to event definition}

\subsection{Sensitivity to event definition}

Now we use the 3 time series described in Sect. 3. It seems that the distributions of event energies $E$ obtained by definitions 1 (a) $\langle 1\rangle$

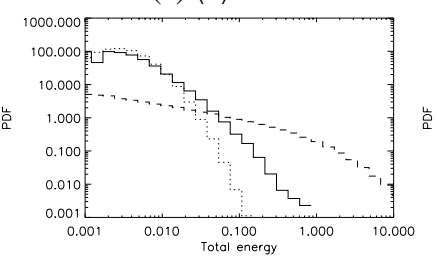

(b) $\langle 2\rangle$

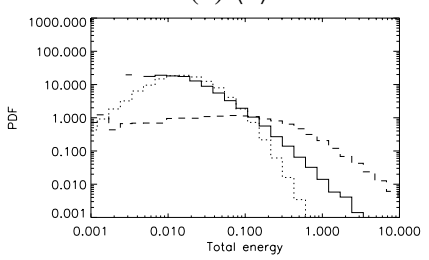

(c) $\langle 3\rangle$

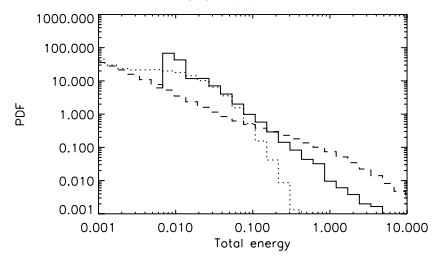

Fig. 11. a) Total energy distributions for events determined by definitions 1 (peaks; dotted line), 2 (threshold; plain line), and 3 (maxima in wavelet time-scale space; dashed line), for time series $\langle 1\rangle$. b) Same figure for time series $\langle 2\rangle$. c) Same figure for time series $\langle 3\rangle$. All plots have the same scale.

(a) $\langle 1\rangle$

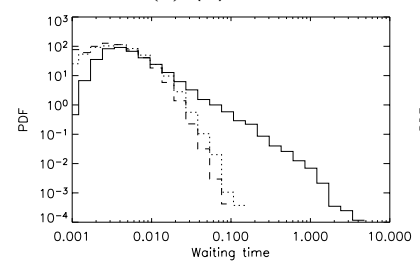

(c) $\langle 3\rangle$

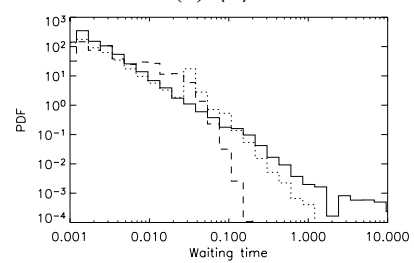

Fig. 12. a) Waiting-time distributions for events determined by definitions 1 (peaks; dotted line), 2 (threshold; plain line) and 3 (maxima in wavelet time-scale space; dashed line), for time series $\langle 1\rangle$. b) Same figure for time series $\langle 2\rangle$. c) Same figure for time series $\langle 3\rangle$. All plots have the same scale.

and 3 are closer to the power-law obtained by definition 2 in the case of time series $\langle 3\rangle$ (Fig. 11c) than in the case of time series $\langle 1\rangle$ (Fig. 11a). The waiting-time distributions (Fig. 12) display the same behavior. In general, distributions obtained from higher-intermittency time series seem to be less sensitive to the definition of an event than low-intermittency time series.

\subsection{Sensitivity to threshold, for definition 2 (threshold)}

In the case of events defined by a threshold (like definition 2), the slope of event energy histograms may depend on the threshold. Here we choose different values of $\epsilon_{\text {thr }}$ between 0 and $\bar{\epsilon}+5 \sigma_{\epsilon}$ where $\bar{\epsilon}$ is the time series average $(\bar{\epsilon}=1)$ and $\sigma_{\epsilon}$ is the standard deviation shown in Table 1 for each of the time series. As a result, the number of events (Fig. 13e) is 1 when the threshold is $\epsilon_{\mathrm{thr}}=0$ (the whole time series is one event); it increases to a maximum, attained between $\bar{\epsilon}$ and $\bar{\epsilon}+\sigma_{\epsilon}$, 

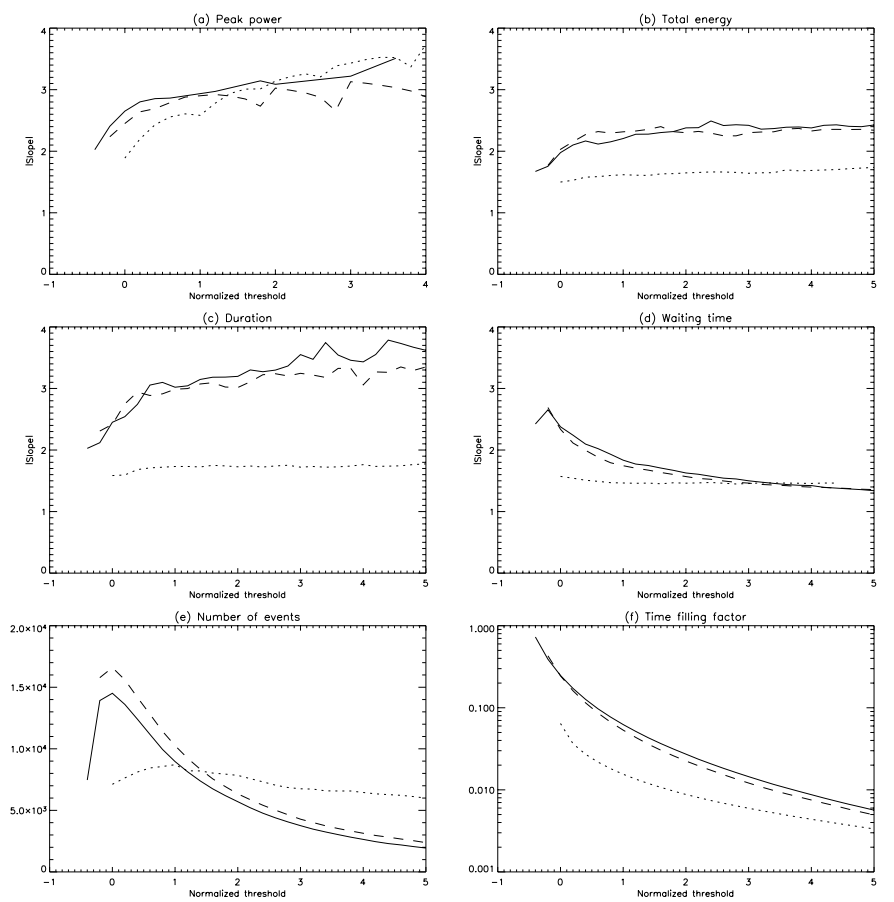

Fig. 13. Slope of the peak power a), total energy b), duration c), and waiting time d) distributions, for events defined by definition 2 (threshold), as a function of the normalized threshold $\left(\epsilon_{\mathrm{thr}}-\bar{\epsilon}\right) / \sigma_{\epsilon}$. The plain, dashed and dotted lines correspond to time series $\langle 1\rangle,\langle 2\rangle$ and $\langle 3\rangle$ respectively. e) Number of events detected as a function of the normalized threshold. f) Proportion of the duration contained in events, as a function of the normalized threshold.

depending on the time series characteristics; then it decreases (ultimately, the number of events is 0 when $\epsilon_{\mathrm{thr}}>\epsilon_{\max }$, where $\epsilon_{\max }$ is the maximum value of the time series).

Figure 13 shows the power-law slope of the histograms of $P, E, T$, and $\tau_{\mathrm{w}}$ as a function of the normalized threshold $\left(\epsilon_{\mathrm{thr}}-\bar{\epsilon}\right) / \sigma_{\epsilon}$. In general, time series $\langle 1\rangle$ and $\langle 2\rangle$, which come from the same simple shell-model and which are less intermittent than the time series $\langle 3\rangle$, follow a similar path. (a) The distributions of peak dissipation power $P$ have a slope $\approx 2$ for a low threshold $\epsilon_{\mathrm{thr}}$, and become steeper when $\epsilon_{\mathrm{thr}}$ increases. The slope for time series $\langle 3\rangle$ is slightly more sensitive to $\epsilon_{\text {thr }}$ than the other time series. (b) The slope of the distributions of energy $E$ also increase with $\epsilon_{\mathrm{thr}}$, except for time series $\langle 3\rangle$, for which it is almost constant. (c) The statistics of the durations $T$ exhibit the same features as the statistics of $E$. (d) On the contrary, the slope of the distributions of the waiting times $\tau_{\mathrm{w}}$ decreases when $\epsilon_{\mathrm{thr}}$ increases. Again, it is almost constant for time series $\langle 3\rangle$. (f) The proportion of the time series duration contained in events decreases when the threshold increases. This decrease is stronger for the slightly intermittent time series.

Time series $\langle 3\rangle$ seems to be the least sensitive to the value of $\epsilon_{\mathrm{thr}}$. Note that by using thresholds expressed as a function of $\sigma_{\epsilon}$ instead of absolute thresholds, we have taken care of the fact that the deviations of time series $\langle 3\rangle$ are larger than for the other time series.

\section{Discussion}

We have investigated the dependence on the definition of "events" of the statistics of events obtained from an energy dissipation time series. Not very surprisingly, the statistics of peak power, energy content, duration and waiting times of events differ when different definitions are used.

Especially for low-intermittency time series and for waiting-time distributions, power-law distributions are recovered only when a threshold is used, either when searching for events (definition 2) or after having searched for events by another means (definitions 1.2 and 3.1). It is also interesting to note that the waiting-time distribution, which is used to test the Poissonian nature of the flaring process (Wheatland et al. 1998; Lepreti et al. 2001; Wheatland \& Litvinenko 2002), can have a power-law or an exponential tail, depending on the definition of events.

For observational studies, where the smallest events are averaged over the line of sight and the spatial and temporal steps, some of the intermittency is lost. In this case we need to use a definition which gives statistics as close as possible to the statistics of the underlying (non-averaged) signal (which is intermittent enough for event statistics to be almost independent of their definition). The definitions using a threshold seem to be adequate from this point of view. The presence of noise in observations also gives strong support to this kind of definition. However, these definitions also have drawbacks, in particular the difficulty of choosing a threshold for a non-stationary time series.

Other definitions like 3 which uses wavelets can have interesting properties, separating simultaneous events at different scales but the smallest events obtained by this means seem to be not significant. Alternatively, events could be defined iteratively from the time-scale plane: the first event is defined by the overall maximum of the time-scale plane, the corresponding wavelet is subtracted from $\epsilon(t)$, a new time-scale plane is computed, and this process is repeated to find each of the next events. The Local Intermittency Measure (LIM: Farge 1990) could perhaps also be used for this purpose. However, these ideas have not been investigated yet and an iterative definition may be computationaly very expensive compared to the other definitions.

Let us now return to the motivation behind the determination and discussion of event properties and statistics for coronal physics. There are two main reasons for these studies, essentially related to bridging the gap between observable time and spatial scales and the sub-resolution physics. On the one hand, we would like to understand whether analogous physical processes, namely flares, conserve scale-invariant properties at unobservable scales and are responsible for the existence of the quiet corona as we know it. On the other, one would like to link, as far as possible, large-scale physical models and numerical simulations to the observations without reproducing in detail the microcosm of a single small-scale event (although this may be desirable and necessary for the largest-scale manifestations, such as for example the Bastille day flare). Rather, one would do this by comparing global statistical properties. 
When searching for the answer to the first question, one must use an event definition that conserves the total energy in the signal, as one is searching for a quantitative confirmation (again, much care is needed, since the average corona exists to some extent precisely because we are incapable of observing fluctuations at sufficiently small energy and time-scale, i.e. it is by definition a background). In the second case however, where there are undoubtedly large differences between numerical models and observations in the richness of the physics and dynamical range, one must be careful to analyze events in the way most appropriate to glean characteristic properties of the fluctuations and turbulence at the available scales. Hence one must go beyond simply the energy distributions of events and analyze other characteristic features such as anisotropy of the spectra, intermittency and higher order structure functions of the fields.

Acknowledgements. The authors acknowledge partial financial support from the PNST (Programme National Soleil-Terre) program of INSU (CNRS) and from European Union grant HPRN-CT-200100310 (TOSTISP network). E. Buchlin thanks the French-Italian University for travel support. The authors thank Jean-Claude Vial, Loukas Vlahos and Jean-François Hochedez for useful discussions.

\section{References}

Aschwanden, M. J., \& Charbonneau, P. 2002, ApJ, 566, L59

Aschwanden, M. J., Tarbell, T. D., Nightingale, R. W., et al. 2000, ApJ, 535, 1047

Bak, P., Tang, C., \& Wiesenfeld, K. 1988, Phys. Rev. A, 38, 364

Biskamp, D. 1994, Phys. Rev. E, 50, 2702
Boffetta, G., Carbone, V., Giuliani, P., Veltri, P., \& Vulpiani, A. 1999, Phys. Rev. Lett., 83, 4662

Boffetta, G., de Lillo, F., \& Musacchio, S. 2002, Phys. Rev. E, 66, 066307

Buchlin, E., Aletti, V., Galtier, S., et al. 2003, A\&A, 406, 1061

Buchlin, E., Velli, M., \& Galtier, S. 2004, in Proc. Conf. SOHO 15, Coronal Heating, ESA SP-575

Crosby, N. B., Aschwanden, M. J., \& Dennis, B. R. 1993, Sol. Phys., 143,275

Dmitruk, P., Gómez, D. O., \& DeLuca, E. E. 1998, ApJ, 505, 974

Einaudi, G., Velli, M., Politano, H., \& Pouquet, A. 1996, ApJ, 457, L113

Farge, M. 1990, in Topological Fluid Dynamics, ed. H. Moffat (Cambridge University Press)

Frick, P., \& Sokoloff, D. 1998, Phys. Rev. E, 57, 4155

Georgoulis, M. K., Velli, M., \& Einaudi, G. 1998, ApJ, 497, 957

Giuliani, P., \& Carbone, V. 1998, Europhys. Lett., 43, 527

Gledzer, E. B. 1973, Sov. Phys. Dokl., 18, 216

Gloaguen, C., Léorat, J., Pouquet, A., \& Grappin, R. 1985, Physica D, 17,154

Hudson, H. S. 1991, Sol. Phys., 133, 357

Lepreti, F., Carbone, V., \& Veltri, P. 2001, ApJ, 555, L133

Lu, E. T., \& Hamilton, R. J. 1991, ApJ, 380, L89

Nigro, G., Malara, F., Carbone, V., \& Veltri, P. 2004, Phys. Rev. Lett., 92, 194501

Pearce, G., Rowe, A. K., \& Yeung, J. 1993, A\&AS, 208, 99

Sanz, J. L., Herranz, D., \& Martínez-Gónzalez, E. 2001, ApJ, 552, 484

Wheatland, M. S., \& Litvinenko, Y. E. 2002, Sol. Phys., 211, 255

Wheatland, M. S., Sturrock, P. A., \& McTiernan, J. M. 1998, ApJ, 509,448

Yamada, M., \& Ohkitani, K. 1987, J. Phys. Soc. Jpn, 56, 4210

Yamada, M., \& Ohkitani, K. 1988a, Progr. Theo. Phys., 79, 1265

Yamada, M., \& Ohkitani, K. 1988b, Phys. Rev. Lett., 60, 983 